
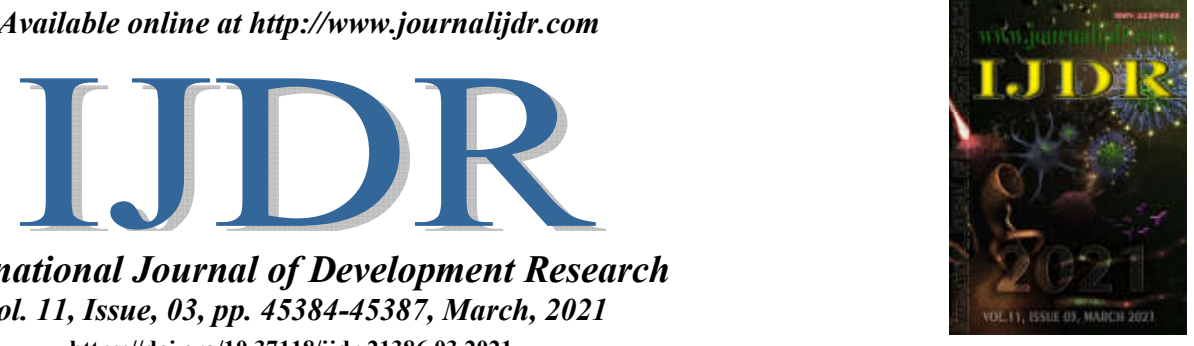

\title{
A DOR FANTASMA E SUAS PRINCIPAIS ABORDAGENS TERAPÊUTICAS: REVISÃO DE LITERATURA
}

\section{Wilde José Cardoso Tanajura Filho*1, Kleber Alves Gomes², Mariane Costa Santos de Tavares ${ }^{3}$, João Pedro Gomes Saback ${ }^{4}$, Yasmin dos Santos Felisberto ${ }^{4}$, Tayná Fagundes Rodrigues ${ }^{4}$ and João Matheus Barbosa de Lima Pinho4}

${ }_{1}^{1}$ Discente do Curso de Graduação em Medicina Faculdades Santo Agostinho, Vitória da Conquista - BA, Brasil; ${ }^{2}$ Biólogo. Mestre em Genética e Biologia Molecular pela Universidade Estadual de Santa Cruz, Doutor em Biotecnologia pela Universidade de São Paulo - USP. Docente do Curso de Graduação em Medicina Faculdades Santo Agostinho, Vitória da Conquista -BA, Brasil; ${ }^{3}$ Fisioterapeuta. Especialista em Fisioterapia Respiratória e

Terapia Intensiva pelas Faculdades Integradas Pitágoras, Discente do Curso de Graduação em Medicina Faculdades Santo Agostinho, Vitória da Conquista - BA, Brasil; ${ }^{4}$ Discente do Curso de Graduação em Medicina Faculdades Santo Agostinho, Vitória da Conquista - BA, Brasil

\section{ARTICLE INFO}

\section{Article History:}

Received $08^{\text {th }}$ January, 2021

Received in revised form

$27^{\text {th }}$ January, 2021

Accepted $11^{\text {th }}$ February, 2021

Published online $26^{\text {th }}$ March, 2021

\section{Key Words:}

Membro fantasma. Pseudomelia.

Manejo da dor. Neuralgia.

\begin{abstract}
A síndrome dolorosa do membro fantasma, ou "dor do membro fantasma" caracteriza-se como um quadro álgico potencialmente debilitante, com difícil manejo clínico e farmacológico, que acomete pacientes previamente amputados. Embora de etiologia não totalmente esclarecida, acredita-se que a dor neuropática apresentada seja decorrente de associação de mecanismos centrais e periféricos das vias aferentes da dor, bem como de uma reorganização cortical e sensitiva. O objetivo deste estudo foi identificar e descrever o manejo farmacológico e não farmacológico para abordagem da síndrome dolorosa do membro fantasma em pacientes amputados. Trata-se de uma revisão integrativa de literatura, cuja busca por estudos se deu nas bases vinculadas à Medical LiteratureAnalysis and Retrieval System Online (MEDLINE), Literatura Latino-americana e do Caribe em Ciências da Saúde (LILACS) e Scientific Electronic Library Online(SciELO). Os descritores em ciências da saúde (DECS) utilizados para busca foram: membro fantasma (pseudomelia), manejo da dor eneuralgia. Não há consenso na literatura sobre um tratamento medicamentoso definitivo para abordagem da dor do membro fantasma. A utilização de antagonistas do receptor N-metil-D-aspartato (NMDA), como a ketamina, são referidos como pioneiros na tentativa de reduzir a sensibilização neuronal. $\mathrm{O}$ uso de opioides, sobretudo associado com outros agentes como anticonvulsivantes e antidepressivos tricíclicos também tem apresentado bons resultados. A maior parte dos estudos referem que o tratamento da dor neuropática em pacientes amputados ainda apresenta lacunas significativas, sendo que a abordagem multidisciplinar é referida como primordial. Dentre as técnicas não medicamentosas destacam-se a terapia do espelho, uso de sistemas de realidade virtual, bem como a estimulação magnética transcraniana repetitiva. Foram citados ainda a acupuntura e crioablação nervosa como estratégias no manejo da dor do membro fantasma.
\end{abstract}

*Corresponding author:

Wilde José Cardoso Tanajura Filho

Copyright (C) 2021, Wilde José Cardoso Tanajura Filho et al. This is an open access article distributed under the Creative Commons Attribution License, which permits unrestricted use, distribution, and reproduction in any medium, provided the original work is properly cited.

Citation: Wilde José Cardoso Tanajura Filho, Kleber Alves Gomes, Mariane Costa Santos de Tavares, João Pedro Gomes Saback, Yasmin dos Santos Felisberto, Tayná Fagundes Rodrigues and João Matheus Barbosa de Lima Pinho, 2021. "A dor fantasma e suas principais abordagens terapêuticas: revisão de literatura", International Journal of Development Research, 11, (03), 45384-45387.

\section{INTRODUCTION}

Associação Internacional para Estudos da Dor (IASP) em sua revisão mais atualizada define dor como uma "experiência sensitiva e emocional desagradável, associada, ou semelhante àquela associada, a uma lesão tecidual real ou potencial".
Entende-se ainda que a dor é uma "experiência pessoal”, e que poderá ser influenciada por fatores sociais, psíquicos, e biológicos em diferentes graus (RAJA, CARR, et al., 2020). Conceitua-se amputação como a retirada total ou parcial de algum membro do corpo humano. Além dos aspectos psicossociais, a perda de um membro ou de parte dele irá impactar de forma dramática a 
funcionalidade, aquisição de novas habilidades, e consequentemente a qualidade de vida do indivíduo(VELASCO, GARCIA, et al., 2015). No cenário brasileiro e no mundo apesar de não se ter um valor preciso da incidência de amputações, estima-se globalmente 2,8 a 43,9 casos por 100000 habitantes/ ano e no Brasil 13,9 por 10000 habitantes/ ano(COIMBRA e MEDEIROS, 2018). As Diretrizes de Atenção à Pessoa Amputada indicam que, em 2011, 49.165 pessoas tiveram algum membro corporal amputado. Calcula-se que $85 \%$ das amputações sejam de membros inferiores, sendo o percentual registrado em 2011 de $94 \%$ dentre todas as amputações realizadas pelo SUS (BRASIL, 2013). A dor fantasma é uma sequela consequente da amputação de membros que pode se apresentar de diversas formas, com início imediato pós-amputação, ou iniciar-se após meses ou anos decorridos da retirada do membro. Fundamentada em fatores psíquicos e fisiológicos ela não possui etiologia bem definida, e as hipóteses etiológicas mais aceitas baseiam-se no resultado da reorganização cortical e nas mudanças somatossensoriais após a amputação (SOUZA FILHO, OLIVEIRA, et al., 2016). Tal dor é descrita como uma dor neuropática, em que a sensação dolorosa se dá muitas vezes distante do local em que ocorreu a lesão, ou doença do sistema nervoso (DESANTANA, PERISSINOTT, et al., 2020).

A dor do membro amputado, ou Síndrome Dolorosa do Membro Fantasma é descrita pela literatura como uma sequela comum da amputação, ocorrendo em aproximadamente $85 \%$ dos pacientes, e pode se apresentar como dor no coto da amputação, dor no membro fantasma ou sensação do membro fantasma. A dor fantasma, foco deste estudo, é a sensação dolorosa que se refere ao membro perdido, sendo mais comum em membros superiores ou inferiores, embora haja também relatos da ocorrência após amputação de outras regiões corporais como mama, pênis, língua, reto, dentre outros(JACON e IEMBO, 2020). A dor fantasma pode se apresentar em diferentes padrões, podendo ser contínua, ou intermitente, apresentar períodos do dia de maior amplitude, ou ainda manifestar-se em intervalores aleatórios. Enquanto alguns pacientes apresentam ciclos de dor com duração de segundos, outros podem sentir a sintomatologia dolorosa por minutos ou horas, embora raramente tenha duração superior à três dias entre seus ciclos (ALMEIDA, 2017). A amputação acaba apresentando-se como uma condição clínica de difícil abordagem terapêutica por sua grande diversidade sintomatológica, limitações funcionais e repercussões psíquicas. Nos últimos anos diversos estudos buscam propor métodos eficazes para o diagnóstico e tratamento da dor fantasma. Em tal contexto o objetivo deste estudo foi identificar e descrever o manejo farmacológico e não farmacológico para abordagem da síndrome dolorosa do membro fantasma em pacientes amputados.

\section{MATERIAIS E MÉTODOS}

Sob o aspecto metodológico o estudo se caracteriza como uma revisão integrativa de literatura, de caráter exploratório, e abordagem qualitativa dos dados coletados. Buscou-se então estruturar uma revisão da literatura científica sobre a dor fantasma e seu manejo terapêutico, levando-se em consideração abordagens farmacológicas e não farmacológicas. Foram incluídos artigos originais, disponíveis na íntegra, nos idiomas inglês e português, que abordassem a temática central acima descrita, publicados nos últimos cinco anos (20162021). A busca por estudos se deu nas bases de dados vinculadas à Medical LiteratureAnalysis and Retrieval System Online (MEDLINE), Literatura Latino-americana e do Caribe em Ciências da Saúde (LILACS) e Scientific Electronic Library Online (SciELO). Os descritores em ciências da saúde (DECS) utilizados para busca foram: membro fantasma (pseudomelia), manejo da dor e neuralgia, bem como seus termos correspondentes na língua inglesa. Inicialmente, no processo de seleção dos estudos procedeu-se a leitura dos títulos e resumos, para posterior leitura integral dos estudos selecionados. A análise do material se deu pela técnicas de análise de conteúdo, em que foram analisadas similaridades e abordagens "chave" para compor categorias temáticas para discussão.
Após análise foram estruturadas duas categorias principais: "Abordagem não farmacológica da dor fantasma" e "Manejo farmacológico da dor fantasma". Por se tratar de uma revisão de literatura, baseada em dados secundários o presente estudo foi dispensado da submissão ao Comitê de Ética em Pesquisa de acordo com a Resolução 466/12 do Conselho Nacional de Saúde (CNS), embora todos os preceitos éticos tenham sido respeitados, garantindo a legitimidade das informações, respeito aos direitos autorais, e análise imparcial dos dados coletados na literatura.

\section{RESULTADOS E DISCUSSÃO}

Após busca criteriosa na literatura foram selecionados doze estudos para discussão.

A. Abordagem não farmacológica da dor fantasma: Uma das abordagens mais descritas pela literatura foi denominada como "terapia do espelho". Trata-se de uma técnica aplicada através de um espelho posicionado no plano sagital, entre os membros que se deseja abordar, de maneira que o reflexo do espelho esteja voltado ao membro íntegro. Ao identificar o reflexo do membro sadio os pacientes amputados possuem uma "sensação virtual" de que tem seu membro amputado, o que contribui para uma redução neuromuscular e funcional. No manejo da dor fantasma, acredita-se que a terapia auxilie em uma melhor plasticidade neuronal, com organização de informações somatossensoriais tanto em vias aferentes como eferentes (SOUZA, CARQUEJA e BAPTISTA, 2016). A terapia do espelho foi proposta por Ramachandran e Rogers-Ramachandran em1996, e baseia-se na ideia de que o movimento imaginário do membro amputado poderia auxiliar na reorganização e integração entre propriocepção e funcionalidade neuromuscular. Em estudo randomizado realizado por Finn et al. (2017) os pesquisadores buscaram a partir da utilização da Terapia do espelho melhora da dor fantasma em membros superiores. Houve heterogeneidade na resposta à terapia, de maneira geral, para alguns pacientes a terapia foi útil na redução da dor fantasma, enquanto para outros não houve ganhos. Contudo, em situações de tratamento físico com o espelho coberto verificou-se até piora da dor em alguns pacientes(FINN, PERRY, et al., 2017). Em modelo teórico proposto por Rothgangel et al. (2016) os pesquisadores ressaltam a individualidade da resposta à terapia do espelho. A Terapia foi testada de forma remota, com uso de folhetos explicativos e DVD, terapia do espelho intensa, incluindo sessão de até 3 horas de duração, e terapia do espelho pré-gravada, em que os movimentos do membro não amputado eram gravados em uma projeção espelhada. Todas as intervenções apresentaram resultados favoráveis, com melhora da dor fantasma nos participantes, contudo, os pesquisadores ressaltam a importância da abordagem individualizada, orientações claras e precisas, além da superioridade da orientação presencial em detrimento da orientação remota(ROTHGANGEL, BRAUN, et al., 2016). Wareham e Sparkes (2020) afirmam que até $70 \%$ dos militares amputados sofrem de dor fantasma, de difícil manejo, e que compromete significativamente qualidade de vida e funcionalidade destes. Conforme o estudo, a dor fantasma nos membros geralmente está associada a uma desorganização cortical, associada à prejuízo da lateralidade. $\mathrm{O}$ estudo não encontrou benefícios unânimes da dor, e lateralidade após sessão de TE. Contudo, dentre os 16 participantes, dois relataram melhora na limitação funcional, e redução da dor respectivamente. Diante de tais dados sugere-se outros estudos com número maior de sessões, bem como número mais significativo de participantes (WAREHAM e SPARKES , 2020). Outros recursos não farmacológicos também tem sido relatados para abordagem da dor do membro fantasma. Em ensaio clínico realizado com 21 pacientes com dor fantasma refratária a demais tratamentos foi realizada crioablação nervosa guiada por imagem percutânea, com uma melhora substancial na funcionalidade e redução dos escores de dor. Antes do procedimento os pacientes apresentavam uma média basal do escore álgico de 6,2 com posterior redução para 2,0 nos níveis álgicos(PROLOGO, GILILAND, et al., 2017). A crioablação ou crioneurólise historicamente vem sendo utilizada para manejo da dor em doenças metastáticas. Embora seja um recurso inovador para 
manejo da dor do membro fantasma é consenso na literatura a necessidade de novos estudos, bem como elaboração de protocolos validados para o tratamento(BITTMAN, PETERS, et al., 2018). A acupuntura foi outra técnica mencionada pela literatura para abordagem não farmacológica da dor do membro fantasma. Foram incluídos no estudo quinze pacientes amputados de membros inferiores, submetidos à acupuntura por um período de quatro semanas. Houve melhora na intensidade média da dor, até um mês após as sessões de acupuntura, contudo, o estudo não aborda a associação de outras técnicas ou tratamentos pelos usuários, que possam também influenciar nos resultados obtidos e/ou observados(TREVELYAN, TURNER, et al., 2016).

B. Abordagens farmacológicas da dor fantasma: Dados da literatura apontam que as abordagens farmacológicas da dor em membro fantasma incluem antidepressivos, antagonistas do receptor $\mathrm{N}$-metil-D-aspartato, anticonvulsivantes, opioides, anestésicos, toxina botulínica e calcitonina. Embora o tratamento multimodal seja referido como preferencial, associando técnicas farmacológicas e não farmacológicas, comumente inicia-se a abordagem do paciente com o manejo farmacológico (URITS, SEIFERT, et al., 2019). Embora sejam largamente utilizados a literatura aponta que anticonvulsivantes, opióides e analgésicos para abordagem da dor fantasma geralmente possuem uso prolongado, que podem acarretar dependência química, reações adversas, bem como elevados custos para saúde. Estudo retrospectivo envolvendo 328 pacientes que sofreram amputação de membros aponta que entre os indivíduos que nunca haviam utilizado tais medicações antes da amputação $(n=216$, $66 \%$ ) $20 \%$ passou a utilizar de forma persistente os opioides após o pós-operatório. Evidenciando assim o impacto da dor no membro fantasma, e a dificuldade de seu manejo após a amputação (STEEN, LIRK e SIGURDSSON, 2020). A dor do membro fantasma é referida como refratária à tratamento medicamentoso, mesmo quando os pacientes fazem uso de "coquetéis tradicionais" incluindo opioides fortes, hipnóticos, antidepressivos, analgésicos adjuvantes, anestésicos locais e psicotrópicos. Em caso clínico apresentado, obteve-se melhora sustentada da dor em paciente com dois anos de sintomatologia dolorosa incapacitante. Utilizou-se buprenorfina / naloxona sublingual, que possui mecanismos multimodais que contribuem para abolição das respostas álgicas. Diante dos resultados obtidos os pesquisadores sugerem a introdução de buprenorfina / naloxonacomo uma escolha precoce de opioide para abordagem da dor do membro fantasma(SREJIC e BANIMAHD, 2021). Diante da insuficiência dos opioides em controlar a dor do membro fantasma comumente são utilizados medicamentos adjuvantes como gabapentina, pregabalina e bupicavaína. Em estudo de caso clínico sugere-se associar a peptinaintranasal durante as crises de dor neuropática em pacientes reflatários às demais medicações. Os pesquisadores referiram que a utilização do spray nasal de fentanil (PecFent $\left.{ }^{\circledR}\right)$ obteve resultados satisfatórios na redução da dor em membro fantasma(HERNÁNDEZ e MONTOSA, 2017). O medicamento já havia sido referido na abordagem da dor crônica oncológica, sobretudo nos episódios de exacerbação álgica enfrentadas por estes pacientes(GUTIÉRREZ e SHAHI, 2015). Em estudo abordando a utilização da ketamina para dor em membro fantasma foi proposta a utilização de ketamina oral três dias antes o procedimento de amputação de membros, e a dor aguda após a amputação. De acordo com os autores a ketamina intravenosa já foi descrita em muitos estudos para reduzir o consumo de opioides no pós-operatório, o que justificaria o uso do fármaco na prevenção da dor do membro fantasma. Três indivíduossubmetidos à amputação eletiva da extremidade inferior foram incluídos no estudo. A primeira dose analgésica de ketamina oral $(1,0 \mathrm{mg} / \mathrm{kg})$ foi administrada uma hora antes da cirurgia, a segunda dose após 8 horas decorridas da primeira medicação. No primeiro dia pós-operatório, os indivíduos receberam ketamina oral $(1,0 \mathrm{mg} / \mathrm{kg})$ três vezes ao dia; e no segundo dia pós-operatório, esta dosefoi reduzida para $0,5 \mathrm{mg} / \mathrm{kg}$ três vezes por dia. Não foram verificados efeitos adversos, representando segurança no uso da ketamina. Acredita-se que o medicamento possa ser útil também para uso doméstico, sobretudo em pacientes com grande limitação funcional pela dor do membro fantasma (BUVANENDRAN, KROIN, et al., 2018).

\section{CONCLUSÃO}

De maneira geral considera-se que o tratamento farmacológico é ainda a primeira escolha para manejo da dor no membro fantasma, sendo utilizados em associação ou isoladamente anestésicos locais, amitriptilina, opioides, antidepressivos tricíclicos, dentre outros.Novas drogas tem sido testadas para associação ao arsenal já em uso, ou em eventos agudos como nos momentos de piora da dor (crise álgica). Outras abordagens não farmacológicas descritas pela literatura incluem a terapia do espelho, acupuntura, uso de neuromodulação não invasiva, estimulação elétrica transcutânea, além de treinamento sensório motor e crioablação nervosa. Contudo, vários estudos referem grande individualidade na apresentação da dor e resposta ao tratamento pelos pacientes, o que dificulta a proposição de protocolos realmente válidos e eficazes. É consenso na literatura a necessidade de uma abordagem multiprofissional e multimodal da dor do membro fantasma como forma de reduzir o quadro álgico, otimizar a funcionalidade, e sobretudo incrementar a qualidade de vida e condição de saúde destes pacientes.

\section{REFERÊNCIAS}

ALMEIDA, I. M. V. M. D. C. E. Dor do Membro Fantasma: revisão bibliográfica da fisiopatologia. Universidade da Beira Interior. Covilhã, p. 43. 2017.

BITTMAN, R. W. et al. Percutaneous Image-Guided Cryoneurolysis. AJR Am J Roentgenol, v. 210, n. 2, p. 454-465, 2018.

BRASIL. Diretrizes de atenção à pessoa amputada. Ministério da Saúde. Brasília, p. 36. 2013.

BUVANENDRAN, A. et al. Oral Ketamine for Acute Pain Management After Amputation Surgery. Pain Med , v. 19, n. 6, p. 06-11, 2018.

COIMBRA, T. L.; MEDEIROS, R. P. Frequencia e fatores determinantes da dor do membro fantasma em pacientes amputados assistidos por um centro de reabilitação situado no centro-oeste do Brasil. Acta Fisiatr, v. 25, n. 1, p. 7-11, 2018.

DESANTANA, J. M. et al. Tradução para a língua portuguesa da definição revisada de dor pela Sociedade Brasileira para o Estudo da Dor. BrJP, São Paulo, v. 3, n. 3, 2020.

FINN, S. B. et al. A Randomized, Controlled Trial of Mirror Therapy for Upper Extremity Phantom Limb Pain in Male Amputees. Front Neurol, v. 8, n. 267, 2017.

GUTIÉRREZ, L. C.; SHAHI, P. K. Investigação clínica del fentanilo con pectina intranasal Pesquisaclínica em spray nasal de fentanil pectina. Medicina Paliativa, v. 22, n. 1, p. 57-61, 2015.

HERNÁNDEZ, A. V.; MONTOSA, G. F. ¿Sirven los opioides de liberación inmediata para el dolor del miembro fantasma? Caso clínico. Rev. Soc. Esp. Dolor, v. 24, n. 5, p. 269-272, 2017.

JACON, J. C.; IEMBO, F. Diagnósticos e intervenções de enfermagem em pacientes com amputação e membro fantasma: mapeamento cruzado. Cuid Enferm., v. 14, n. 2, p. 164-171, 2020.

PROLOGO, J. D. et al. Percutaneous Image-Guided Cryoablation for the Treatment of Phantom Limb Pain in Amputees: A Pilot Study. J Vasc Interv Radiol, v. 28, n. 1, p. 24-34, 2017.

RAJA, S. N. et al. The revised International Association for the Study of Pain definition of pain: concepts, challenges, and compromises. Pain, v. 161, n. 9, p. 1976-1982, 2020.

ROTHGANGEL, A. et al. Development of a Clinical Framework for Mirror Therapy in Patients with Phantom Limb Pain: An Evidence-based Practice Approach. Pain Pract, v. 16, n. 4, p. 422-434, 2016.

SOUZA FILHO, L. et al. Tratamento da dor fantasma em pacientes submetidos à amputação: revisão de abordagens clínicas e de reabilitação. Revista Brasileira de Ciências da Saúde, v. 20, n. 03, p. 241-246, 2016.

SOUZA, J. B. D.; CARQUEJA, C. L.; BAPTISTA, A. F. Physical rehabilitation to treat neuropathic pain. Revista Dor, v. 17, p. 8590, 2016. 
SREJIC, U.; BANIMAHD, F. Haunting of the phantom limb pain abolished by buprenorphine/naloxone. BMJ Case Rep, v. 14, n. 2, 2021.

STEEN, T.; LIRK, P. B.; SIGURDSSON, M. L. The demographics of persistent opioid consumption following limb amputation. Acta Anaesthesiol Scand, v. 64, n. 3, p. 361-367, 2020.

TREVELYAN, E. G. et al. Acupuncture for the treatment of phantom limb syndrome in lower limb amputees: a randomised controlled feasibility study. Trials, v. 17, n. 1, p. 519-522, 2016.

URITS, I. et al. Treatment Strategies and Effective Management of Phantom Limb-Associated Pain. Curr Pain Headache Rep, v. 23, n. 9 , p. 64-69, 2019.
VELASCO, A. et al. Dolor fantasma en niños y jóvenes amputados adquiridos: prevalencia y características clínicas. Rehabil Integral, v. 10, n. 1, p. 8-16, 2015.

WAREHAM, A. P.; SPARKES , V. Effect of one session of mirror therapy on phantom limb pain and recognition of limb laterality in military traumatic lower limb amputees: a pilot study. BMJ Mil Health, v. 166, n. 3, p. 146-150, 2020. 